\title{
A novel progressively swarmed mixed integer genetic algorithm for security constrained optimal power flow (SCOPF)
}

\author{
Mithun M. Bhaskar ${ }^{1}$, Srinivas Muthyala ${ }^{2}$ and Maheswarapu Sydulu ${ }^{3}$ \\ ${ }^{1,3 *}$ Department of Electrical Engineering, National Institute of Technology, Warangal, INDIA \\ ${ }^{2 *}$ Infosys Technologies Limited, Mysore, INDIA \\ "Corresponding Author e-mail: mithun.bm@ieee.org, Tel +91-903-2595259
}

\begin{abstract}
This paper proposes a superior Mixed Integer based hybrid Genetic Algorithm (MIGA) which inherits the advantages of binary and real coded Genetic Algorithm approach. The proposed algorithm is applied for the conventional generation cost minimization Optimal Power Flow (OPF) problem and for the Security Constrained Optimal Power Flow problem. Here, the main shortcoming with the conventional Genetic Algorithm, the 'Hamming Cliff' problem is addressed with Mixed Genetic Algorithm, which can overcome issues connected to the continuous search space. The proposed algorithm models the continuous variables using real values and discrete variables using binary values. A novel concept of Progressive filling is also presented here for Mixed Integer GA, which heightens the algorithm. The proposed procedure is compared with many conventional algorithms and validated on a test-bed of standard IEEE 30 bus system with and without valve-point loading effect.
\end{abstract}

Keywords: Genetic Algorithm, Optimization Techniques, Optimal Power Flow, Power System Security.

\section{Introduction}

The restructuring of the electric power industry has brought in a paradigm shift in the real-time control of power grids. Effective dispatch is one of the important control activities in a Power System and Optimal Power Flow (OPF) has been the most significant technique for obtaining minimum cost generation patterns in a Power System together considering the transmission and operational constraints. Classical Optimization techniques have usually considered OPF as minimization of an objective function representing the generation cost and/or the transmission loss. The constraints involved are the physical laws governing the power generation-transmission systems and the operating limitations of the equipment. But, effective Optimal Power Flow is characterized by its high dimensionality and the incomplete domain dependent knowledge of Power System engineers. The first limitation is addressed by numerical optimization procedures based on successive linearization using the first and the second derivatives of objective functions and their constraints as the search directions or by linear programming solutions to imprecise models. The advantages of such methods are existing in their mathematical underpinnings, but disadvantages also exist in the sensitivity to problem formulation, algorithm selection and usually converge to a local minimum. The second limitation, incomplete domain knowledge, precludes also the reliable use of expert systems where rule completeness is not possible. Therefore, an efficient and effective OPF procedure is needed to help the operators to utilize the Power System controls in a realistic and optimal or near optimal manner. Furthermore, when the valve-point loading effect or the prohibited operating zones of generator is considered, conventional techniques can hardly obtain the optimal solution, because these techniques cannot offer greater freedom in objective functions or the types of constraints that may be used.

Dommel and Tinney (1968) has reported a detailed survey on Load flow algorithms, the first tributes for load flows (Carpentair, 1962) and earlier methods were basically of linearized DC load flow models with many approximations. The proposal on secure loading of power system (Wells, 1968) and works on rescheduling with security constraints (Kaltenback and Hajdu, 1971) and (She and Laughton, 1970) were the primary works on power system security constrained optimization, (Alsac and Stott, 1974) in early 1973 proposed a more accurate (earlier methods were DC approximate methods) method to incorporate the steady state security constraints into OPF, which allowed to consider the reactive power and voltage constraints in outage cases. Rodrigues $e t$ 
al. (1994) proposed asynchronous method with dual-simplex relaxation solution for the linearized SCOPF with parallel architecture processing for the preventive mode of SCOPF. (Conforti et al., 1996) proposed Parallel Gradient Distribution (PGD) and Non-Linear Programming based OPF algorithm with (N-1) contingencies with continuous security. Vaahedi et al. (2001) was the pioneer to include the dynamic constraints; voltage stability with the static security constraints viz., the flow and voltage profile during normal and post contingency operations. Ramesh and Xuan (1997) put forward a decomposed form of Contingency Constrained Optimal Power Flow (CCOPF) using Fuzzy Logic where, the minimization of both the base case (pre-contingency) operating cost and of the post-contingency correction times which are conflicting, were accepted as fuzzy goals.

The literatures states that the main constraint was that the problem tends to settle in a global optimum as security constraints are difficult to be included in the line security constraints into fitness function. Lai et al. (1997) proposed a Binary Coded Improved Genetic Algorithm approach for the normal and contingent condition of the system with a simulated circuit outage. (Somasundaram et al., 2004) put forward an evolutionary programming based solution for the SCOPF problem which was superior and robust technique using the first and second derivatives of objective function or constraints and is independent of the nature of the search space. The difficulties due to the 'hamming cliff' problem has been well reported in Zhong et al. (2001)

\section{Problem Formulation}

A load flow method usually determines the state variables such as voltage magnitudes and phase angles of buses except at slack/swing/reference bus, for a given loading condition. Several hundreds of load flow solutions are required to arrive at an optimal power flow solution with enforcement of all equality and inequality constraints.

If the security constraints such as transmission capacity limit $\&$ the bus voltage limits are taken into account, the corresponding OPF is termed as Security Constrained Optimal Power Flow (SCOPF). The generalized fuel cost minimization objective function can be put forward as,

$$
F(x)=\sum_{i=1}^{N_{g}}\left(A_{i}+B_{i} P_{g i}+C_{i} P_{g i}^{2}\right) \$ / h r
$$

The quadratic cost model for generation of power will be utilized and $\mathrm{PG}_{\mathrm{i}}$ is the amount of generation in megawatts at generator 'i' and $\mathrm{Ng}$ is the number of generators. When the valve-point loading effect of thermal units is also taken into consideration, the fuel cost will be of the form

$$
F(x)=\sum_{i=1}^{N g}\left(A_{i}+B_{i} P_{g i}+C_{i} P_{g i}^{2}\right)+\mid d_{i} \sin \left(e_{i}\left(P_{g i-\min }-P_{g i}\right) \mid \$ / h r\right.
$$

where, $A_{i}, B_{i}$ and $C_{i}$ are the cost coefficients of unit $i$. $\mathrm{Ng}$ is the number of generators. The sinusoidal term added to the fuel cost function which models the valve-point effect introduces ripples to heat-rate curve and therefore introducing more local minima to the search space and $d_{i}$ and $e_{i}$ are the value point loading constants, which adds the sinusoidal ripples due to the opening of each steam value in the system.

2.1 Equality Constraints: The equality constraints of the OPF reflect the physics of the power system as well as the desired voltage set points throughout the system. The dynamics of the Power System are enforced through the power flow equations which require that the net injection of real and reactive power at each bus sum to zero.

$$
\begin{aligned}
& P_{g i}-P_{\text {Loadi }}-V_{i} \sum_{j=1}^{n b} V_{j}\left(G_{i j} \sin \delta_{i j}+B_{i j} \sin \delta_{i j}=0\right. \\
& Q_{g i}-Q_{\text {Loadi }}-V_{i} \sum_{j=1}^{n b} V_{j}\left(G_{i j} \sin \delta_{i j}-B_{i j} \cos \delta_{i j}=0\right.
\end{aligned}
$$

2.2 Inequality Constraints: The inequality constraints of the OPF reflect the limits on physical devices in the power system as well as the limits created to ensure system security. Physical devices that require enforcement of limits include generators, tap changing transformers, and phase shifting transformers. Generators have maximum and minimum output powers and reactive powers which add to the inequality constraints.

2.3 Variables: Practically the variables in OPF problem can be divided into two as continuous variables and discrete variables. Continuous variables are generation of generators $\left(\mathrm{P}_{\mathrm{g}}\right)$, Generator bus voltage magnitudes $(\mathrm{V})$ and VAR injection values of SVC. Discrete Variables are transformer tap setting $\left(T_{p}\right)$, VAR injection values of switchable shunt capacitor /reactor $\left(V_{h}\right)$ and phase shifter angle positions. In practical operation, because the reactive power injection of switched shunt device depends on the bus voltage, the specified VAR-injection value of switched shunt device (such as shunt capacitor bank) is difficult to adjust. The solution to the problem is to select the switched shunt admittance as the discrete control associated with the switched shunt devices; it will be capable of obtaining good control level. 
2.4 Hamming Cliff Problem: Conventional modeling of chromosomes in Genetic Algorithm suffers from the severe drawback of taking some large number of mutative steps from an equivalent decimal value to the other. The Electrical Power Flow problem being highly convex, non-linear and consists of continuous and discrete variables (Generator Power and Voltage being continuous and Transformer and shunt settings are discrete), will have a higher probability to settle in a local minima instead of global minima due to the Hamming Cliff Problem. Here in this paper, the novel hybrid approach of using Mixed Integer GA resolves this as the variables are modeled using its most adaptive technique depending on the nature of the behavior itself.

2.5 Security Constraints: by adding the security constraints in the objective function, OPF problem is expressed as a mixed integer non-convex programming. Contingency analysis is carried out by Inverse Lemma Technique. Herein, one of the lines is selected for outage study to test the security of the system and is compared with the normal conditions.

\section{Proposed Method}

Genetic Algorithm has been fascinating researchers for long and a first credit is given to John Holland. Genetic Algorithm, a stochastic routine, delivers quality solution from a random search space and population, where each generation undergoes transformation using Genetic operators to improve them. Genetic Algorithm transvers multiple peaks in parallel, assuring global solutions and has the advantage of modeling discrete and continuous variables together which is not available with other algorithms. Much variants and developments in Genetic Algorithm has been discussed in (Chaiyaratana and Zalzala, 1997).

The conventional GA suffers from the difficulty in the hamming cliffs associated with certain strings, from which a transition to a neighboring solution (in real space) and requires the alteration of many bits. Hamming cliffs present in a binary coding cause artificial hindrance to a gradual search in the continuous search space. The other difficulty is the inability to achieve any arbitrary precision in the optimal solution. In binary coded GA's, the string length must be chosen a priori to enable GAs to achieve a certain precision in the solution. The more the required precision, then the larger is the string length. For large strings, the population size requirement is also large, thereby increasing the computational complexity of the algorithm. Since a fixed coding scheme is used to code the decision variables, variable bounds must be such that they bracket the optimum values.

Since real parameters are used directly (without any string coding), solving real parameter optimization problems is a step easier when compared to the binary coded GAs. Unlike in the binary coded GAs, decision variables can be directly used to compute the fitness values. Since the selection operator works with the fitness value, any selection operator used with binary coded GAs can also be used in real parameter GA's. Another difficulty arises with the search operators. In the binary coded GA's, decision variables are coded in finite length strings and exchanging portions of two parent strings easier to implement and visualize. Simply flipping a bit to perform mutation is also convenient and resembles a natural mutation event. In real parameter GA's, the main challenge is how to use a pair of real parameter decision variable vectors to a mutated vector in a meaningful manner. As in such cases the term, crossover is not that meaningful, they can be best described as blending operators. However, most blending operators in real parameter GA's are known as crossover operators. In most of the evolutionary algorithms, the final solution depends on the initial generation of population. Keeping the above in view, we proposed a method called 'Successive Filling Approach' (SFA) for initial generation of genetic population, which will guide to better optimal solution with in less number of iterations, which is described in the later sections of this paper.

3.1 Constraint Handling: Constraints are handled by using penalty function approach when and most commonly we encounter while trying to solve a minimization problem is the nonexistence of a feasible solution. Essentially this means that too many constraints have been added to the problem and no solution exists which obeys all of the constraints. One way to avoid this issue is to implement soft inequality constraints in the form of penalty functions. The word "soft" signifies that the constraint is not absolutely enforced. The soft constraint only encourages the solution to meet the constraint by enforcing a penalty if the constraint is not met. In the OPF problem, soft equality constraints are not used, because of the nature of the equality constraints in the OPF problem. The power flow equations cannot be violated as they are imposed by physics, and the generator set points of a Power System are normally not moved around frequently. For the inequality constraints, the penalty functions offer a viable option. Ideally, a penalty function will be very small, near a limit and increase rapidly as the limit is violated more. The quadratic penalty function used here has the ability to control how hard or soft to make the constraint. If an individual $S_{\mathrm{j}}$ is a feasible solution and satisfies all constraints, its fitness will be measure by taking the reciprocal of the fuel cost function else it need to be penalized.

3.2 Successive Filling Approach: It is a new method proposed herein to generate potential initial population before iteration process is initiated. This aspect offers significant reduction in computational burden. In any heuristic technique, the number of iterations taken for convergence condition depends upon the initial generation of population and with a superior initial population, the problem tends to converge in a fewer number of iterations and consumes low computational time.

3.3 Fitness Evaluation: PF-MIGA has two arithmetic crossover schemes which are employed to treat the continuous \& discrete variables and to produce two types of substrings, respectively. The typical value of probability of crossover $\left(\mathrm{P}_{\mathrm{c}}\right)$ used here is about 
90\%. The arithmetic crossover schemes of the continuous/discrete variables are as follows. For discontinuous variables, the crossover is applied with a probability $\mathrm{P}_{\mathrm{c}}$ in the range of 0.8 to 0.9 . Here, the objective is a minimization problem and hence the fitness is the reciprocal of evaluated objective function. Proper penalty factors are to be selected judiciously by the operator as operators experience \& suitability of the selected penalty factors will have significant influence on the speed of convergence.

\section{Simulation and Validation}

The effectiveness of the MIGA method is tested against two other real coded Algorithms PSO \& DE using standard IEEE-30 bus test system in terms of solution quality and evolutionary computing efficiency. The network consists of 6 Generator buses, 21 load buses \& 41 lines, of which 4 lines are due to tap setting transformers. Buses 10, 12, 15, 17, 20, 21, 23, $24 \& 29$ have been selected as shunt compensation buses. The total load on the network is 283.4 MW. Five generator active power outputs, six generator-bus voltage magnitudes, four transformer tap-settings \& nine shunt susceptances, which counts to 24 control variables.

The gene length for unit active power outputs is 12 bits, generator voltage magnitude is 8 bits, and both of them are treated as continuous control variables. As the transformer tap settings can take 17 discrete values each one is encoded using 5 bits $\&$ the step size is 0.0125 p.u. The bus shunt susceptance can take 6 discrete values each one is encoded using 3 bits, \& the step size is 0.01 p.u. (on system MVA basis). Thus, the total string length tends to be 155 .

The established population size is 60 with a uniform crossover probability of 0.9. The string length is 155 bits, mutation and elitism probability $\left(\mathrm{P}_{\mathrm{e}}\right)$ of $0.05 \& 0.2$ respectively with a scaling factor of 0.6 . PSO, the swarm size is 60 and the size of particle is 24. The acceleration constants are $\mathrm{C} 1=\mathrm{C} 2=2.05$, inertia Weight $(\mathrm{W})$ is 1.2 and Constriction Factor $(\mathrm{K})$ is 0.7295 . For DE, the population size is 60 ; vector length is 24 , Scaling factor of 0.8 with a crossover rate of 0.7 .

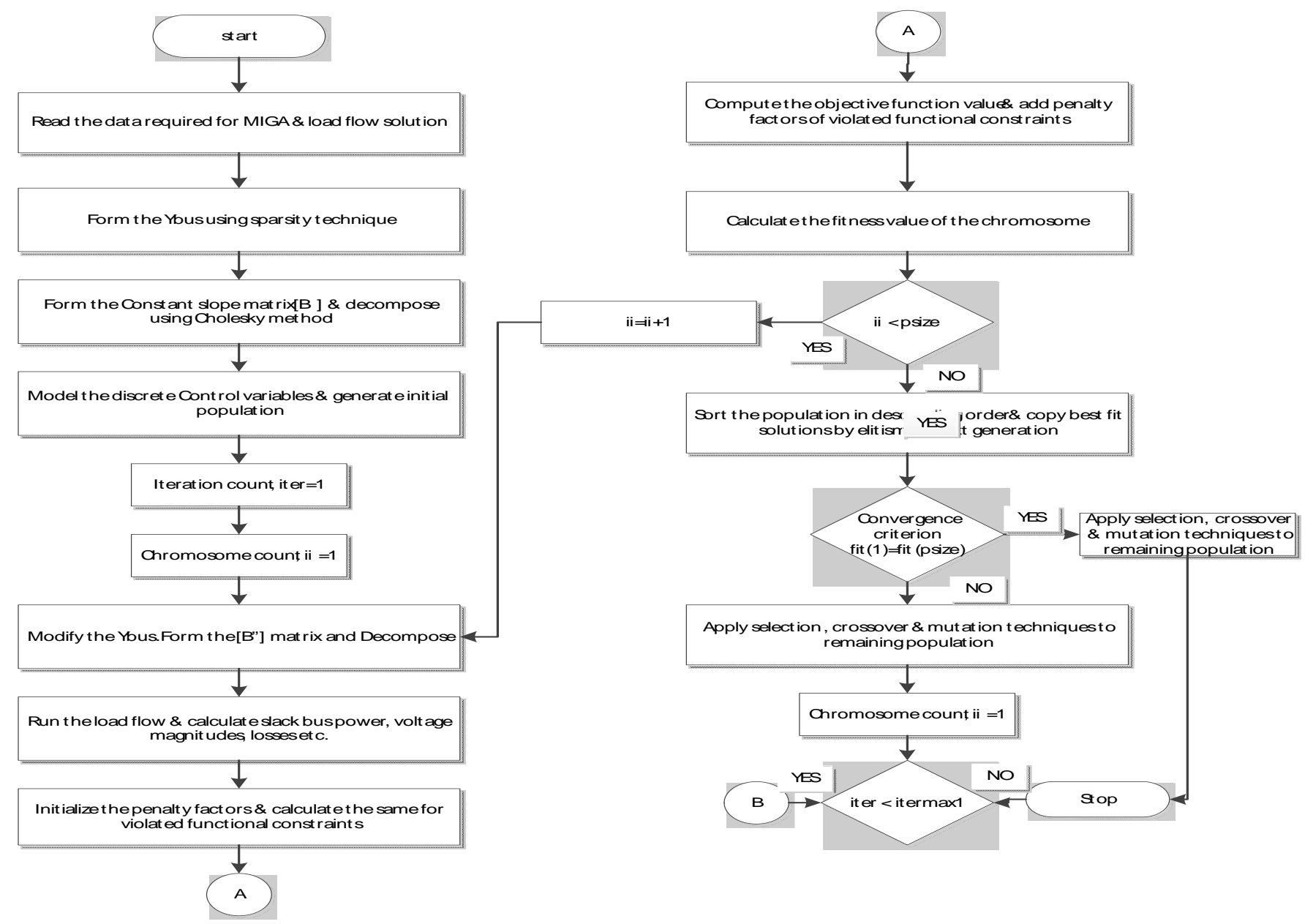

Figure 1. Flow Chart of the Proposed Algorithm 
Table 1. Control Variables with and without Value Point Loading and Contingency state

\begin{tabular}{|c|c|c|c|c|c|c|c|c|c|c|c|c|c|}
\hline \multirow{3}{*}{$\begin{array}{c}\text { Control } \\
\text { Variable }\end{array}$} & \multirow{3}{*}{$\begin{array}{l}\text { Base } \\
\text { Case }\end{array}$} & \multicolumn{12}{|c|}{ ALGORITHM } \\
\hline & & \multicolumn{3}{|c|}{ SGA } & \multicolumn{3}{|c|}{ PSO } & \multicolumn{3}{|c|}{ DE } & \multicolumn{3}{|c|}{ PF-MIGA } \\
\hline & & $\begin{array}{c}\text { WO } \\
\text { VPL }^{*}\end{array}$ & $\mathrm{~W} \mathrm{VPL}^{*}$ & $\mathrm{WCA}^{+}$ & $\begin{array}{l}\text { WO } \\
\text { VPL }^{*}\end{array}$ & W VPL ${ }^{*}$ & $\mathrm{WCA}^{+}$ & $\begin{array}{l}\text { WO } \\
\text { VPL }^{*}\end{array}$ & W VPL ${ }^{*}$ & $\mathrm{WCA}^{+}$ & $\begin{array}{c}\text { WO } \\
\text { VPL }^{*}\end{array}$ & W VPL ${ }^{*}$ & $\mathrm{WCA}^{+}$ \\
\hline $\begin{array}{c}\text { Slack } \\
\text { Bus }\end{array}$ & - & 175.99 & 189.479 & 168.38 & 176.5 & 177.252 & 169 & 177.85 & 190.574 & 168.715 & 178.68 & 175.751 & 168 \\
\hline $\begin{array}{c}\mathbf{P G}_{2} \\
(\mathbf{M W})\end{array}$ & 80.0 & 49.34 & 20.176 & 48.615 & 48.83 & 25.487 & 48.5 & 48.47 & 20 & 48.163 & 48.631 & 26.869 & 48.87 \\
\hline $\mathbf{P G}_{5}$ & 50.0 & 21.93 & 49.068 & 23.846 & 21.13 & 31.483 & 21.8 & 20.78 & 40.486 & 20.934 & 21.936 & 31.661 & 22.92 \\
\hline $\mathbf{P G}_{8}$ & 20.0 & 22.96 & 10.433 & 29.304 & 20.27 & 18.22 & 26.5 & 20.66 & 10 & 26.044 & 21.409 & 12.194 & 25.01 \\
\hline $\mathbf{P G}_{11}$ & 20.0 & 12.78 & 10.762 & 12.882 & 12.37 & 14.783 & 15.7 & 10.00 & 10 & 16.031 & 10.00 & 16.462 & 14.35 \\
\hline $\mathbf{P G}_{13}$ & 20.0 & 12.10 & 12.602 & 12.786 & 12.80 & 12.501 & 14.4 & 14.77 & 12 & 14.833 & 12.00 & 17.331 & 16.74 \\
\hline $\mathrm{VG}_{1}(\mathrm{pu})$ & 1.0 & 1.05 & 1.05 & 1.05 & 1.05 & 1.05 & 1.05 & 1.05 & 1.05 & 1.05 & 1.05 & 1.05 & 1.05 \\
\hline $\mathrm{VG}_{2}$ & 1.0 & 1.01 & 1.005 & 1.002 & 1.044 & 1.034 & 1.04 & 0.95 & 0.95 & 1.076 & 1.041 & 1.019 & 1.026 \\
\hline $\mathrm{VG}_{5}$ & 1.0 & 1.09 & 1.021 & 1.049 & 1.043 & 0.993 & 0.99 & 1.09 & 1.1 & 1.092 & 1.035 & 1.062 & 1.011 \\
\hline $\mathbf{V G}_{8}$ & 1.0 & 1.04 & - & 1.09 & 1.0 & - & 1.07 & 1.10 & - & 1.1 & 0.95 & - & 0.996 \\
\hline $\mathrm{VG}_{11}$ & 1.0 & 1.08 & 1.046 & 0.956 & 1.02 & 1.032 & 1.04 & 1.05 & 0.97 & 1.05 & 0.95 & 1.052 & 0.995 \\
\hline $\mathrm{VG}_{13}$ & 1.0 & 1.02 & 0.956 & 0.979 & 1.01 & 1.062 & 1.05 & 0.98 & 1.1 & 0.951 & 0.95 & 1.007 & 1.033 \\
\hline$T_{6,9}(p u)$ & 1.0 & 0.96 & 0.963 & 0.988 & 0.9 & 0.9 & 0.9 & 0.90 & 0.9 & 0.9 & 0.90 & 0.9 & 0.9 \\
\hline$T_{6,10}$ & 1.0 & 1.05 & 1.025 & 0.925 & 1.1 & 1.1 & 1 & 1.08 & 1.1 & 1.1 & 1.013 & 1.05 & 1.0 \\
\hline$T_{4,12}$ & 1.0 & 1.012 & 1.063 & 0.988 & 1.0 & 1.025 & 0.98 & 0.93 & 1.05 & 0.963 & 0.963 & 1.075 & 1.025 \\
\hline $\mathbf{T}_{27,28}$ & 1.0 & 1.02 & 1.038 & 1.038 & 1.025 & 1.05 & 1.06 & 1.01 & 1.063 & 1.088 & 0.938 & 0.988 & 1.0 \\
\hline $\mathrm{S}_{10}(\mathrm{pu})$ & 0.00 & 0.02 & 0.02 & 0.03 & 0.02 & 0.01 & 0.03 & 0.12 & 0.02 & 0.06 & 0.03 & 0.04 & 0.06 \\
\hline$S_{12}$ & 0.00 & 0.03 & 0.02 & 0.02 & 0.03 & 0.05 & 0.03 & 0.06 & 0.12 & 0.01 & 0.06 & 0.08 & 0.08 \\
\hline$S_{15}$ & 0.00 & 0.02 & 0.05 & 0.02 & 0.05 & 0.04 & 0.03 & 0.02 & 0.02 & 0.01 & 0.06 & 0.1 & 0.03 \\
\hline$S_{17}$ & 0.00 & 0.01 & 0.01 & 0.01 & 0.03 & 0.03 & 0.06 & 0.06 & 0.02 & 0.01 & 0.01 & 0.06 & 0.06 \\
\hline$S_{20}$ & 0.00 & 0.02 & 0.01 & 0.02 & 0.04 & 0.06 & 0.03 & 0.08 & 0.12 & 0.06 & 0.02 & 0.1 & 0.08 \\
\hline$S_{21}$ & 0.00 & 0.04 & 0.02 & 0.05 & 0.04 & 1.032 & 0.04 & 0.06 & 0.97 & 0.06 & 0.01 & 1.052 & 0.04 \\
\hline$S_{23}$ & 0.00 & 0.02 & - & 0.02 & 0.03 & - & 0.02 & 0.08 & - & 0.04 & 0.05 & - & 0.02 \\
\hline$S_{24}$ & 0.00 & 0.05 & 0.03 & 0.05 & 0.02 & 0.03 & 0.03 & 0.06 & 0.12 & 0.04 & 0.04 & 0.1 & 0.05 \\
\hline $\mathbf{S}_{29}$ & 0.00 & 0.04 & 0.04 & 0.03 & 0.01 & 0.05 & 0.05 & 0.06 & 0.1 & 0.04 & 0.02 & 0.02 & 0.08 \\
\hline $\begin{array}{l}\text { Fuel } \\
\text { Cost }\end{array}$ & - & 802.81 & 1302.97 & 816.58 & 801.58 & 1301.92 & 814.92 & 801.06 & 1301.95 & 812.27 & 801.114 & 1301.89 & 816.98 \\
\hline
\end{tabular}

Table 2. Comparison of Algorithms for Best Optimized Condition

\begin{tabular}{|c|c|c|c|c|c|c|c|c|c|c|c|c|}
\hline \multirow{2}{*}{ TECHNIQUE } & \multicolumn{3}{|c|}{ FUEL COST } & \multicolumn{3}{c|}{ LOSSES } & \multicolumn{3}{c|}{ TIME /ITER } & \multicolumn{3}{c|}{ ITERATIONS } \\
\cline { 2 - 12 } & $\begin{array}{c}\text { WO } \\
\text { VPL }^{*}\end{array}$ & $\begin{array}{c}\mathrm{W} \\
\text { VPL }^{*}\end{array}$ & WCA $^{+}$ & $\begin{array}{c}\text { WO } \\
\text { VPL }\end{array}$ & W VPL $^{*}$ & WCA $^{+}$ & $\begin{array}{c}\text { WO } \\
\text { VPL }^{*}\end{array}$ & $\begin{array}{c}\text { W } \\
\text { VPL }^{*}\end{array}$ & WCA $^{+}$ & $\begin{array}{c}\text { WO } \\
\text { VPL }\end{array}$ & $\begin{array}{c}\text { W } \\
\text { VPL }^{*}\end{array}$ & WCA $^{+}$ \\
\hline SGA (WO-VP) & 802.359 & 1302.97 & 816.58 & 9.60 & 9.121 & 12.41 & 0.488 & 0.82 & 0.31 & 40 & 24 & 64 \\
\hline PSO & 802.667 & 1301.92 & 814.92 & 9.59 & 8.870 & 12.47 & 1.737 & 1.81 & 0.48 & 70 & 86 & 78 \\
\hline DE & 801.114 & 1301.95 & 812.27 & 9.54 & 9.166 & 12.32 & 1.236 & 1.43 & 0.74 & 46 & 23 & 53 \\
\hline PF-MIGA & 800.801 & 1301.89 & 816.98 & 9.23 & 8.825 & 12.53 & 0.620 & 0.72 & 0.48 & 37 & 21 & 43 \\
\hline
\end{tabular}

WO-VPL: without Value Point Loading

"WVPL: with Value Point Loading ${ }^{+}$WCA: with contingency analysis 

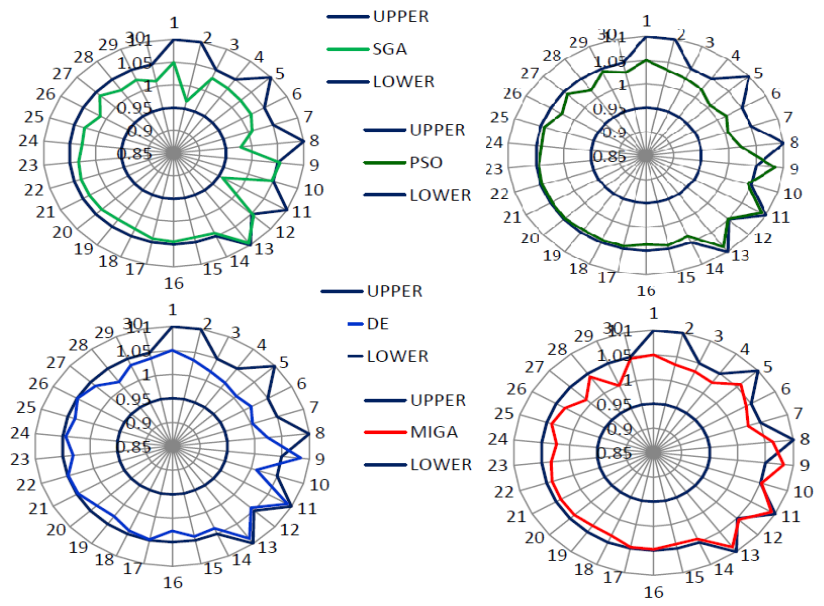

Figure 3. Voltage Profile in PF-MIGA during Contingency

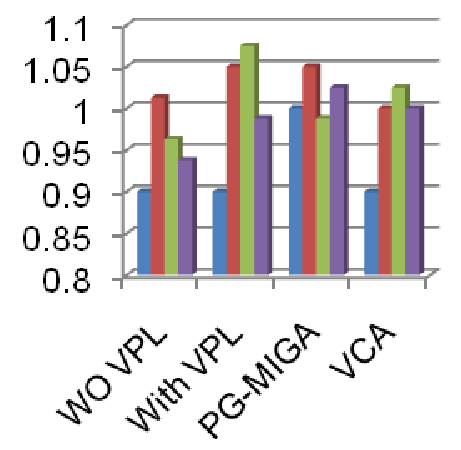

$\because \mathrm{T} 6,9(\mathrm{pu})$

- T6,10

$\because \mathrm{T} 4,12$

- $\mathrm{T} 27,28$

Figure 4. Transformer Tap Settings on various Conditions.

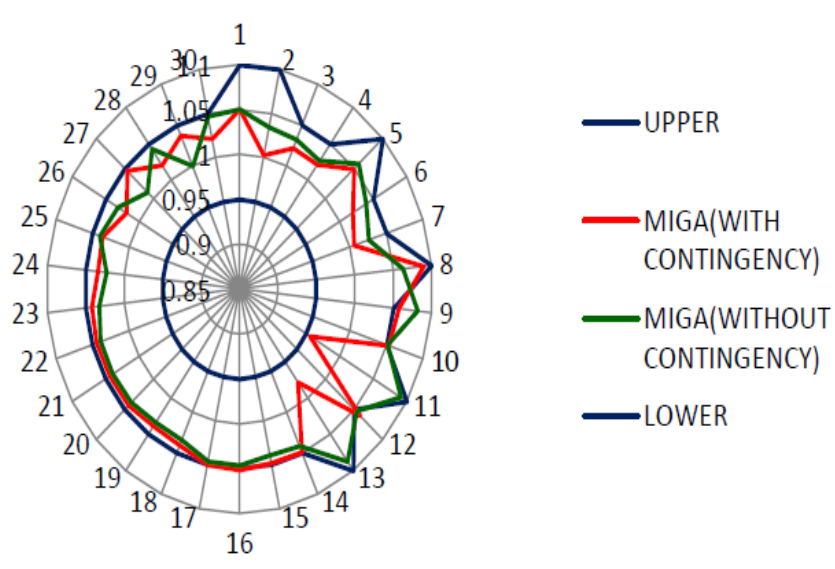

Figure 2. Voltage Profile using SGA, PSO, DE and PF-MIGA.

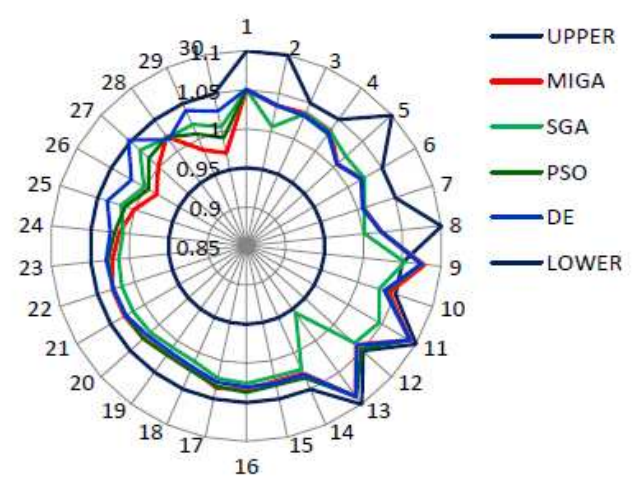

Figure 5. Voltage Profile (with VPL) in SGA, DE, PSO and PF-MIGA

It is observed that the total number of iterations taken for the problem to converge is more when we perform contingency analysis. At the same time the fuel cost $\&$ the corresponding total losses are also increased. Table I reports the details of the control variables using SGA, PSO, DE and MIGA on different states like with and without value point loading and on contingency analysis. It is found that using MIGA the fuel cost for normal condition, with value point loading and with Contingency analysis were $801.114 \$, 1301.89 \$$ and $816.98 \$$ respectively. Table II compares the performance of MIGA with others on Fuel Cost, Computation time, Losses etc and it is found that PF-MIGA is 2.3 times faster than other algorithms in case of contingency analysis and 3.5 times faster in case of normal power flow without value point loading effect. Fig. 1 illustrates the complete process flow chart, Fig. 2 illustrates the bus voltage profile in various algorithms, Fig. 3 illustrates the convergence characteristics in normal cases, Fig. 4 exemplifies the voltage profile of MIGA in contingency and normal conditions, Fig. 5 demonstrates the effect on tap settings in various settings like normal power flow with and without value point loading effect and during contingency analysis. Fig. 6 shows the voltage profile of PF-MIGA with valve point loading and using various algorithms.

\section{Conclusions}

An approach has been made in this paper to develop a new algorithm for the security constrained optimal power flow. The new proposed algorithm offers the flexibility to model the continuous variable using real values and discrete variables using the binary coding and an innovative approach in the initial generation is adopted. The algorithm is found to be much suitable for Contingency analysis and offers 3.5 times faster solution than other methods. The proposed method is compared with other conventional algorithms like Differential evolution, Genetic algorithm and Particle swarm optimization for proving the supremacy. 


\section{References}

Alsac O. and Stott, B. 1974. Optimal load flow with steady state security, IEEE Trans. on Power Apparatus and Systems, Vol. PAS-93, pp. 745-751.

Carpentier J. 1985. Optimal power flows: uses, methods and developments, IFAC Symposium on planning and operation of Electric Energy Systems. (Invited paper)

Chaiyaratana. N and Zalzala, A.M.S., 1997. Recent developments in evolutionary and genetic algorithms: theory and applications.

Genetic Algorithms in Engineering Systems: Innovations and Applications, pp. 270-277.

Conforti, D., Menniti, D., Sorrentino, N., Verde, P., Testa, A., 1996. Optimal load-flow with N-1 steady-state security via high performance computing, Electrotechnical Conference - MELECON '96, 8th Mediterranean, Vol.2, No..2, pp.873-879.

Dommel H. W. and Tinney. W. F., 1968. Optimal load flow solutions. IEEE Transactions on Power Apparatus and Systems, Vol. PAS-87, pp. 1866-1876.

Kaltenbach, J.C. and Hajdu, L.P., 1971. Optimal corrective rescheduling for power system security, IEEE Trans. Power App. and Syst., Vol. PAS-90, No. 2, pp 843-851.

Lai,. L.L., Ma. J. T., Yokoma. R. and Zhao.M., 1997. Improved genetic algorithms for optimal power flow under both normal and contingent operation states. Electrical Power \& Energy System, Vol. 19, pp. 287-292.

Ramesh. V. C., and Xuan Li, 1997. A fuzzy multi-objective approach to contingency constrained OPF, IEEE Trans. Power Syst., Vol. 12, No. 3, pp.1348-1354.

Rodrigues, M., Saavedra, O.R., Monticelli, A., 1994. Asynchronous programming model for the concurrent solution of the security constrained optimal power flow problem, IEEE Transactions on Power Systems, Vol.9, No.4, pp.2021-2027.

Somasundaram.P., Kuppusamy.K., and Devi. R. P. K., 2004. Evolutionary programming based security constrained optimal power flow. Electric Power System. Research, No. 72, pp. 137-145.

Vaahedi. E., Mansour. Y., Fuches C., Granville. S., Latore. M., Hamadanizadeh. H., 2001.Dynamic security constrained optimal power flow/VAr planning, IEEE Trans. on Power Systems, Vol. 16, No. 1, pp 38-43.

Wells, D.W., 1968. Method for Economic Secure Loading of a Power System, IEE Proceedings, Vol. 115, No. 8, pp. 1190-1194

Zhong, M. and Sun, S.D. 2001. Genetic Algorithms: Theory and Applications, Beijing Defense Industry.

\section{Biographical notes}

Mithun M. Bhaskar graduated in Electrical Engineering and Specialized in Electrical drives and control from Pondicherry University. Presently he is a doctoral candidate at National Institute of Technology, INDIA. His present interests include Artificial Intelligence, Nature Inspired and Meta-heuristic Technique applications to Electrical Engineering problems like real time control of Power System, Power system Security, Smart Grids and Multilevel inverters.

Srinivas Muthyala received his Bachelor's Degree in Electrical Engineering from Jawaharlal Nehru Technological University, INDIA in the year 2007 and is pursuing Master's Degree in Power Systems Engineering from National Institute of Technology, Warangal, INDIA. His Areas of interest include Artificial Intelligence \& Meta heuristic technique applications in Power System, FACTS Controllers and Power System Security.

Maheswarapu Sydulu received his B.Tech in Electrical Engineering, Specialized in Power Systems in masters and received Ph.D in 1978,1980 and in 1993 respectively from National Institute of Technology, Warangal, INDIA. Presently he is working as Professor and Dean of the same Institute. His areas of interest include Real Time power system operation and control, Artificial Intelligence techniques applications in Power Systems, Distribution system studies, Economic operation, Reactive power planning and management. Dr. Maheswarapu is member of various organizations including IEI (India), IEE, ISTE etc and is a reviewer of various Journals.

Received September 2010

Accepted November 2010

Final acceptance in revised form December 2010 